\title{
Free Energy of a Large Finite Ideal Bose System
}

\author{
Artur Hahn
}

\author{
(Z. Naturforsch. 28a, 14-22 [1973]; received 2 October 1972)
}

\begin{abstract}
The following problem is investigated: How large must an ideal Bose-system be to allow for the approximation of the finite system free energy by the thermodynamic limit. The obvious condition that the energy separations between the single-particle states must be small compared to $k T$ is necessary but is not sufficient near the critical concentration. Instead, for this case additional stronger conditions apply due to the singularity of the thermodynamic free energy at the critical point which is caused by contributions of the lowest-energy single-particle states. These conditions are derived and the relevancy of the results is emphasized for a generalized theory of metastability in co-operative systems.
\end{abstract}

\section{Problem and Results}

A characteristic finite "cube-shaped" ideal Bose System of Volume $V$ and hence "linear dimension" $V^{1 / s}$ may be defined in terms of Bose number operators $b_{\boldsymbol{k}}{ }^{+} b_{\boldsymbol{k}}$ by the Hamiltonian

$$
\hat{H}=\sum_{\boldsymbol{k}} \varepsilon_{\boldsymbol{k}} b_{\boldsymbol{k}}{ }^{+} b_{\boldsymbol{k}}
$$

where the single particle energies

$$
\varepsilon_{\boldsymbol{k}}=D \boldsymbol{k}^{2}
$$

correspond to standing waves-type excitations of wave vectors

$\boldsymbol{k}=\pi V^{-1 / s} \boldsymbol{x} ; \quad \boldsymbol{x}=\left(\varkappa_{1}, \varkappa_{2}, \varkappa_{3}\right) ; \quad \varkappa_{i}$ integers $\geq 0$.

The following question will be investigated in this paper: What are the conditions, under which the variation of the finite system free energy

$$
f_{V}(L)=-k T V^{-1} \log \operatorname{tr}\left\{\hat{P}_{\mathrm{L}} \exp (-\hat{H} / k T)\right\}
$$

with density $L / V=\varrho^{1}$ may be approximatively replaced by the thermodynamic limit

$$
f(\varrho)=\lim _{\substack{V \rightarrow \infty \\ L / V \rightarrow 0}} f_{V}(L) ?
$$

In (4) $\hat{P}_{\mathrm{T}}$ is the projection operator onto the subspace with $L$ bosons excited.

According to the definition (4),

$$
Z(L)=\exp \left[-V f_{V}(L) / k T\right]
$$

Reprint requests to Prof. Dr. A. HAHN, Institut für Werkstoffe der Elektrotechnik, Ruhr-Universität Bochum, D-4630 Bochum-Querenburg, Postfach 2148.

1 Throughout this investigation we are interested only in the behaviour of the free energy as a function on density with the temperature held fixed. For this reason we only is the partition function of the canonical ensemble corresponding to a closed system. It has carefully to be distinguished, for the finite system, from the partition function of the grand-canonical ensemble corresponding to an open system for which the investigation dealt with in this paper would take a much easier form.

The thermodynamic free energy (5) of the ideal Bose gas with single particle energy spectrum (2) is well known ${ }^{2,3}$ as a function of density and temperature: At fixed $T$ Bose condensation occurs at a critical density

$$
\varrho_{\mathrm{c}}=\pi^{3 / 2} 2^{-3} \zeta(3 / 2) V_{T}^{-1} .
$$

Here $\zeta(z)$ is the Riemann zeta-function and

$$
V_{T}=\left(D \pi^{2} / k T\right)^{3 / 2}
$$

is a characteristic volume. For the condensed system $\varrho>\varrho_{\mathrm{c}}$ the thermodynamic free energy is independent of $\varrho$, whereas for the non-condensed system $\varrho<\varrho_{\text {c }}$ it is a monotonically decreasing downwards-convex function of $\varrho$ given by

$$
f(\varrho)=-k T\left[\varphi_{\infty}\left(\mu^{*}\right)-\varrho \mu^{*}\right],
$$

$\varphi_{\infty}(\mu)=-\int_{0}^{\infty}(\pi / 2) \varkappa^{2} \mathrm{~d} \varkappa V^{-1} \log [1-\exp (\mu-\varepsilon k / k T)]$,

and by the relation defining $\mu^{*}$ in terms of the original independent variables $T$ and $\varrho$ :

$$
\left(\mathrm{d} \varphi_{\infty}(\mu) / \mathrm{d} \mu\right)_{\mu=\mu^{*}=\varrho} .
$$

This relation defines $\mu^{*}$ as a negative quantity for $\varrho<\varrho_{\text {c }}$

indicate the independent variable $L$ or $\varrho$, respectively, in the notation for thermodynamic functions writing $f_{V}(L)$ for example instead of $f_{V}(T, L)$.

2 R. H. Fowler and H. Jones, Proc. Cambridge Phil. Soc. 34, 573 [1938]. 
In the integral (10) $\varepsilon_{k}$ has to be understood as a function of the continuous variable $x / V^{1 / 3}$. The inter gral may be evaluated by series expansion of the logarithm with the result ${ }^{3}$ :

$$
\Psi_{\infty}\left(,(1)=\pi^{3 / 2} 2^{-3} V_{T^{-1}} g(5 / 2,, \text { (1) } .\right.
$$

Here $g(n, \mu)$ is defined by

$$
g(n,, u)=\sum_{l=1}^{\infty} l^{-n} e^{l ! n} .
$$

From this definition one obtains the relation

$$
\partial g(n, ! \prime) / \partial ! \prime=g(n-1, \prime \prime)
$$

which, for example, allows to write (11) more explicitly by inserting (12) and performing the differentiation.

The finite system free energy $f_{V}(V \cdot \varrho)$ is only defined for discrete values $\varrho=L / V$, with $L$ integer, of the argument. Hence the separation of these values is $V^{-1}$. Throughout this investigation we are interested in the case of such large systems only, that the energy separations of the low energy (long-wave length) excitations are small compared to $k T$. The lowest $(\boldsymbol{k} \neq 0)$ excitation energy

$$
\varepsilon_{100}=D \pi^{2} V^{-2 / 3}
$$

may serve as an order of magnitude guess for these energy separations. Hence we may formulate this condition as

$$
\varepsilon_{100} / k T \ll 1
$$

or, in terms of the characteristic volume (8), as

$$
V \gg V_{T} \quad(16 \mathrm{~b}) \quad \text { or } \quad V^{-1} \ll \varrho_{\mathrm{c}}
$$

equivalently.

If one wants approximately to replace $f_{V}(V \varrho)$ by the thermodynamic limit $f(\varrho),(16)$ certainly constitutes a necessary condition. However, in addition to (16) one has to postulate $\varrho$ to be sufficiently different from the critical density $\varrho_{\mathrm{c}}$ in order to comply with the postulate that the variation of $f_{V}\left(V_{\varrho}\right)$ upon small variations of $\varrho$ is correctly described by the derivatives of $f(\varrho)$ with respect to $\varrho$. This is not surprising since $Q=Q_{\mathrm{c}}$ is a singular point of the thermodynamic free energy. This result to be derived in later sections is as follows:

Supplementary to (16) one of the conditions

3 A. Münster, Statistische Thermodynamik, SpringerVerlag, Berlin 1956, \12.7. or

$$
\begin{aligned}
& \varrho_{c}-\varrho \gg k T /\left(V \varepsilon_{100}\right) \\
& \varrho-\tilde{Q}_{c} \gg k T /\left(V \varepsilon_{100}\right)
\end{aligned}
$$

is sufficient to ensure that $f_{V}(0 V)$ is approximated by $f(0)$ in this sense.

Here $\tilde{Q}_{c}$ is a characteristic density depending on $T$ and system size $V$, which is given by $(56)$ and $(57 \mathrm{a}, \mathrm{b})$ below. $\tilde{Q}_{\text {. }}$ is close to $Q_{\text {c }}$ for a large system and tends to $Q_{c}$ in the thermodynamic limit $V \rightarrow \infty$.

It is only under one of these additional conditions (17a) or (18a) that one may use the simple analytical resulis for the thermodynamic free energy as an approximation for the finite system free energy defined in (4).

Making use of (7), (8), and (15) and neglecting unimportant numerical factors, one may equivalently express conditions (17a) and (18a) as

and

$$
\left(V / V_{\mathrm{T}}\right)^{1 / 3} \gg \varrho_{\mathrm{c}} /\left(\varrho_{\mathrm{c}}-\varrho\right) \text { for } \varrho<\varrho_{\mathrm{c}},
$$

$$
\left(V / V_{\mathrm{T}}\right)^{1 / 3} \gg \varrho_{\mathrm{c}} /\left(Q-\tilde{\varrho}_{\mathrm{c}}\right) \text { for } \varrho>\tilde{Q}_{\mathrm{c}}
$$

respectively. In this form the postulate for the magnitude if $V$ is clearly seen to be more stringent than (16b) for small values of $\varrho-\varrho_{c} \mid \varrho_{c}$. Relations (17) and (18) constitute the central result of this investigation. They will be proved in Sections III and IV.

\section{Motivation for this Investigation}

Our interest in the finite Bose system free energy defined by (4) stems from the fact, that this quantity seems to play a central role in the theory of life-time of certain metastable states in co-operative systems very near the limit of stability. Actually the problem investigated here arose during a previous investigation by the author ${ }^{4}$ of the long-time relaxational behavior of ferromagnetic materials after a sudden change of the external magnetic field, the so-called magnetic after-effect. The fundamental process governing this effect is the thermal fluctuation of the state vector of the coupled magnetic system accross barriers of the free energy separating different metastable states in a finite volume of the material. Although small on a macroscopic scale of, say, $\mathrm{cm}$, this volume in any case contains a very large number of coupled atomic spins and hence the relevant processes are fluctuations in the

4 A. Hahn, Z. Naturforsch. 27a, 905 [1972]. 
occupation numbers of collective modes of longwave-length low-energy spin-wave type.

An adequate idealization of the system in the neighbourhood of the metastable state seems possible on the basis of the observation that metastability in such magnetic systems is associated with a removal of spatial degeneracy of an isotropic exchange Hamiltonian by small additional anharmonic forces. In terms of spin-wave theory, spatial degeneracy is reflected by the property $\varepsilon(\boldsymbol{k}=0)=0$ for the energy of the $k=$ 0 spin wave mode. Let $n_{\boldsymbol{k}}$ be the occupation number of spin-wave state $\boldsymbol{k}$ and $L=\sum_{\boldsymbol{k}} n_{\boldsymbol{k}}$ the deviation from the metastable state of the magnetization. The simplest way to introduce into a spin-wave model additional forces which constitute a metastable state in the vincinity of $\left\langle n_{0}\right\rangle=0$ is to introduce a gap in the spinwave spectrum and to represent the anharmonicity of these forces, leading to a free energy barrier at some small $L$, by a linear dependence on magnetization $L$ of the gap energy $A$. Hence in this approximation the all-important long wave-length spin-waves have energies

with

$$
\varepsilon_{\boldsymbol{k}}=A+D \boldsymbol{k}^{2}
$$

$$
A=A(L)=A_{0}-B L / V
$$

and $A_{0}$ and $B$ positive quantities indenpendent of $L$. In this approximation the total free energy of the finite coupled spin system is given by

$$
F(L)=A_{0} L-B L^{2} / V+V f_{V}(L)
$$

with $f_{V}(L)$ determined by (4).

One easily finds out that $F(L)$ has a minimum at some finite $L$ and a maximum at some higher value of $L$ for sufficiently large $A_{0}$. Thus (21) constitutes a model of a metastable state stabilized by a free energy barrier against small deviations.

It is just in this context that the problem investigated in this paper enters the theory of stability of metastable states in the magnetic case. Of course, for this particular problem the quantities $A_{0}$ and $B$ in (20) and (21) have a special physical meaning. In particular, $A_{0}$ has to be assumed a linear function of the external magnetic field $H^{4}$ :

$$
A_{0}=\text { const }\left(H_{\mathrm{co}}-H\right) \text {. }
$$

5 R. Landauer in: J. I. Budnick and M. P. Kawatra (editors), Dynamical Aspects of Critical Phenomena, Gordon and Breach, New York 1972, p. 369.
Hence metastability can be switched to instability by variation of the external field which is quite natural to postulate for a reasonable theory of metastability. In fact in the after-effect problem one is interested in the case of "very weak" stability since only energy barriers of several $\mathrm{kT}$ order of magnitude are surmounted by thermal fluctuations within measurable time.

One of the aimes of the present investigation is to prove some mathematical details which where used without proof in the previous paper ${ }^{4}$ to derive asymptotic results for a special magnetic system and to discuss the range of parameters to which these results apply. However, the point should be stressed here that the simple model described above might be well suited to describe weak metastability in co-operative systems on a more general base. For example a quite similar situtation is to be expected along the hysteresis loop of a ferroelectric material for those parts of the volume the polarization direction of which is very near the limit of stability. As another example considerations starting from (19) - (21) might apply to a type of metastability or bistability in active electronic circuits with non-linear devices of the kind discussed by LANDAUER ${ }^{5}$. In contrast with the foregoing examples, the metastable state in these systems is not a thermodynamic equilibrium state but a stationary state with finite energy dissipation. The similarity between these both types of metastability was stressed by Pytte and Thomas ${ }^{6}$ for the special case of a Gunn diode near the transition to the negative resistance regime.

In all these cases weak metastability, that is the region of transition to instability, is characterized by the frequency of some elementary excitation of the system going to zero. While in the ferroelectric case this "soft mode" is a long wave-length optical phonon it is, according to Pytte and Thomas, a plasma oscillation in the case of the Gunn instability.

As soon as one is interested in the stability of such sytems against thermodynamic fluctuations in some finite partial volume one may expect to meet a problem similar to the ferromagnetic one outlined above.

Concluding this review of a general background we emphasize the following point: the structure of metastable states in real co-operative systems is, in

- E. Pytte and H. Thomas, Phys. Rev. Letters 20, 1167 and 1466 [1968]; Phys. Rev. 179, 431 [1968]. 
general, rather complex. For example,we may think of a Bloch wall pinning mechanism in a ferromagnetic material. For this reason, results of the theory are of particular interest which do not depend on the finer details of the structure and on the values of secondary parameters determining it but on the general analytic behavior of the free energy function and on primary well-known parameters only. Fortunately in the magnetic case there exists such a result for sufficiently large Volume $V$ as was shown in reference ${ }^{4}$. This result concerns the magnetic viscosity coeffient $S_{v}$ defined by the well-known formula?

$$
M(t)=M_{0}+\chi_{\text {irr }} S_{\mathrm{v}} \log t
$$

for the long-time magnetization relaxation function, where $\chi_{\text {irr }}$ is the irreversible susceptibility. The result is, that for large volume $V$ the viscosity coefficient $S_{\mathrm{v}}$ depends only on the spin-wave dispersion coefficient $D$ and not at all on the other parameters $A_{0}$ and $B$ entering (21). This result is of great practical importance since the parameters $A_{0}$ and $B$ are strongly model-dependent and badly known in actual cases whereas $\mathrm{D}$, on the other hand, is a rather well-defined and structure-independent quantity. While the way leading to this result cannot be described here and instead reference must be made to the original paper one may expect that in other cases results may be obtained of similar simplicity on the basis of the more general and less specific properties of a metastable state. In fact this seems the only way along which one might hope to obtain results from theory of some generality.

\section{The Non-Condensed System}

The discussion of the problem outlined in Section I is different for different sign of $\varrho-\varrho_{\mathrm{c}}$. The case of the "condensed system" $\varrho>\varrho_{c}$ will be investigated in section IV. Here we suppose $\varrho<\varrho_{\mathrm{c}}$.

We follow the well-known Darwin-Fowler method of steepest descent. According to this scheme we introduce the generating function

$$
\exp \{V \varphi(z)\}=\prod_{\boldsymbol{k}}\left\{1-z \exp \left(-\varepsilon_{\boldsymbol{k}} / k T\right)\right\}^{-1}
$$

7 for reviews see for example: W. F. BRown in: R. E. Burgess (editor), Fluctuation Phenomena in Solids, Academic Press, N. Y. 1965, pp. 37-78; H. KRON-
The $L^{\text {th }}$ coefficient in the Taylor series expansion of this function

$$
\exp \{V \varphi(z)\}=\sum_{i=} Z(L) z^{L}
$$

is just the partition function corresponding to $f_{V}(L)$ and defined in (6).

Hence introducing the function

$$
\begin{gathered}
\psi(z)=q(z)-(L / V) \log z, \\
\exp [V \psi(z)]=z^{-L} \exp [V \psi(z)]
\end{gathered}
$$

one may represent $Z(L)$ by the contour integral

$$
Z(L)=(2 \pi i)^{-1} \oint(\mathrm{d} z / z) \exp [V \psi(z)],
$$

with the contour including the pole of the integrand at $z=0$ and excluding the poles at $z=z_{\boldsymbol{k}}=\exp (\varepsilon \boldsymbol{k}$ ! $k T$ ) on the real axis.

In Fig. 1 the positions of poles in the complex $z-$ plane are marked schematically by crosses. Moreover the saddle-point of $\exp \left[V_{\psi}(z)\right]$ on the real axis is

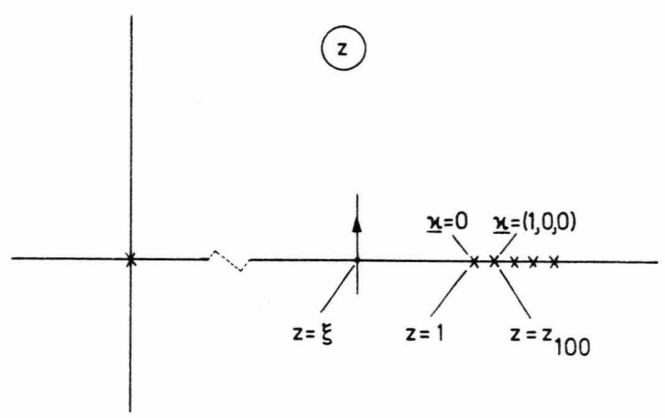

Fig. 1. Poles (x) and saddle-point $\xi$ of $\exp [V \psi(z)]$ in the complex $z$-plane. The poles on the positive real axis are in a one-to-one correspondence with the single-particle energies. Those corresponding to the ground- and first excited states are marked by their quantum numbers $x$. The case treated in this section is characterized by $1-\xi$ $\gg z_{100}-z_{0}$ whereas the opposite order-of-magnitude relation, $1-\xi \ll z_{100}-z_{0}$, underlies the discussion in Section IV. In any case $z_{100}-z_{0} \ll 1$ is supposed. The figure schematically represents the first of the two cases. In this case the main contribution to the integral (27) stems from a small region around $\xi$. The contor of integration is indicated in this region.

indicated. Let its position be

$$
z=\xi=e^{\mu *}, \mu^{*}<0 .
$$

MÜLLER, Nachwirkung in Ferromagnetika, SpringerVerlag, Berlin 1968, Chapts. 2.2 and 4.2. 
Then $\xi$ or $\mu^{*}$ are given by

$$
[\mathrm{d} / \mathrm{d} z\{\exp [V \psi(z)]\}]_{z=\xi}=0
$$

or, equivalently, by

$$
[\mathrm{d} \psi(z) / \mathrm{d} \log z] \log z=\mu^{*}=0 .
$$

According to (26a) this may also be written as

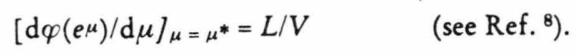

Inserting the definition (24) of $\varphi(z)$ into this equation and performing the differentiation, one obtains the most familiar form of the saddle-point condition, namely

$$
L / V=V^{-1} \sum_{\boldsymbol{k}}\left\{\exp \left(\varepsilon_{\boldsymbol{k}} / k T-\mu^{*}\right)-1\right\}^{-1} .
$$

The method of steepest descend now proceeds in lowest order by expanding $\psi(z)$ in (27) in powers of the deviation $z-\xi$ from the saddle-point

$$
\psi(z)=\psi(\xi)+\frac{1}{2} \psi^{\prime \prime}(\xi)(z-\xi)^{2}+\ldots
$$

Only terms up to second order are retained and the integration is extended to $\xi \pm i \infty$. One obtains ${ }^{9}$

$$
Z(L)=e^{V \psi(\xi)}\left[2 \pi V \xi^{2} \psi^{\prime \prime}(\xi)\right]^{-\frac{1}{2}} .
$$

For later use we denote here the relation

$$
\xi^{2} \psi^{\prime \prime}(\xi)=\left[\mathrm{d}^{2} \varphi\left(e^{\mu}\right) / \mathrm{d} \mu^{2}\right]_{\mu=\mu^{*}}
$$

which is easily obtained from (26a), (28), and (30).

Taking the logarithm of (34) and using (6) and (26a) one has

$$
\begin{aligned}
f_{V}(L)=-k T\left\{\varphi(\xi)-(L / V) \mu^{*}-(2 V)^{-1}\right. \\
\\
\left.\log \left[2 \pi V \xi^{2} \psi^{\prime \prime}(\xi)\right]\right\}
\end{aligned}
$$

with

$$
\varphi\left(\mathrm{e}^{\mu}\right)=-V^{-1} \sum_{\boldsymbol{k}} \log \left\{1-\exp \left(\mu-\varepsilon_{\boldsymbol{k}} / k T\right)\right\}
$$

from (24), and $\mu^{*}=\log \xi$ determined by (31), (37) as a function of $L$.

Hence comparison of Eqs. (36), (37), and (31) with (9), (10), and (11) respectively makes clear that the thermodynamic limit is a good approximation to the finite particle free energy as soon as the parameters of the model have values which allow

1) to replace the discrete sum in (37) by the integral

\footnotetext{
8 We have decided not to give different symbols to the quantities $\mu^{*}$ appearing in Sect. I and here in order to emphasize that, $\varrho$ being fixed, $\mu^{*}$ in both cases is the same functional of the functions $\varphi_{\infty}(\mu)$ and $\varphi\left(e^{\mu}\right)$,
}

(10) via the substitution

$$
V^{-1} \sum_{\boldsymbol{k}} \rightarrow \int_{0}^{\infty}(\pi / 2) x^{2} \mathrm{~d} x / V
$$

2) to neglect the third term on the right-hand side of (36),

3) to restrict saddle-point integration to the lowest order term at all.

These three conditions are satisfied if, and only if,

$$
\varepsilon_{100} / k T \ll-\mu^{*}
$$

is true in addition to the weak condition (16a).

(39) represents an additional condition, going beyond (16a) for $-\mu^{*} \ll 1$, that is near the critical point. We restrict discussion to this case which alone is non-trivial. The following discussion will show that (39) in fact implies the approximations 1), 2), 3) one after another.

Approximation 1): That (39) is the appropriate condition for 1) is clear from the observation that the well-known singularity of the thermodynamic free energy at the condensation point $\mu^{*}=0^{-}$stems from the divergency of the corresponding integrals representing the second and higher derivatives of the free energy with respect to $\mu^{*}$ at the lower limit of integration. For example the second derivative of $\varphi_{\infty}\left(\mu^{*}\right)$ diverges for $\mu^{*} \rightarrow 0^{-}$as

$\int_{0}^{\infty} \frac{x^{2} \mathrm{~d} x \exp \left(x^{2}-\mu^{*}\right)}{\left[\exp \left(x^{2}-\mu^{*}\right)-1\right]^{2}}$ i. e. proportional to $\left(-\mu^{*}\right)^{-\frac{1}{2}}$.

Here $x=\sqrt{D} \pi V^{-1 / 3} x$ is introduced for abbrevation.

Apparently close to this singular point the lowest $\boldsymbol{k}$ contributions to the discrete sum and its dervatives play a dominant role. Hence the discrete sum cannot be replaced by an integral despite (16a) as long as $-\mu^{*}$ is too small. The condition of being far enough off the condensation point clearly is (39) as may be seen by inspection of the lowest $-\boldsymbol{k}$ contributions to $\varphi(\xi)$ and its derivatives with respect to $\mu^{*}$.

Approximation 2): In order to estimate the order of magnitude of the terms on the right-hand side of (36) one may use the corresponding quantities in the thermodynamic limit. The first two contributions to the curled bracket are then given by

respectively. However the fact must be kept in mind that $\mu^{*}$ takes different values in both cases since the functions $\varphi_{\infty}(\mu)$ and $\varphi\left(e^{\mu}\right)$ differ.

$\because$ see, for example, Ref. ${ }^{3}, \$ 3.5$. 
$\varphi_{\infty}\left(\mu^{*}\right)-\varrho \mu^{*}=\pi^{3 / 2} 2^{-3} V_{T}^{-1}\left[g\left({ }^{5} / 2, \mu^{*}\right)-\mu^{*} g\left(3 / 2, \mu^{*}\right)\right]$

on account of (12), (11), and (14). The third is

$$
\gamma\left(u^{*}\right)=-(2 V)^{-1} \log \left[2 \pi \cdot \pi^{3 / 2} 2^{-3}\left(V / V_{T}\right) g\left(\frac{1}{2}, u^{*}\right)\right]
$$

according to (35) and, again, (12) and (14).

The contribution (42) to the free energy is not extensive in contrast with the leading term (41) and hence does not survive the thermodynamic limit. For the finite system, however, condition (16b) is not sufficient in order to justify the neglect of $\gamma\left(\mu^{*}\right)$ near $\mu^{*}=$ - O since $g\left(\frac{1}{2}, \mu^{*}\right)$ diverges for $\mu^{* \rightarrow 0}$.

As a reasonable postulate in the case of small values of $-\mu^{*}$ one may require that the variation with $\varrho$ and hence with $\mu^{*}$ must be small for $\gamma\left(\mu^{*}\right)$ as compared to $q_{\infty}\left(\mu^{*}\right)-\varrho \mu^{*}$, i.e.

$$
\left|\mathrm{d} \gamma\left(u^{*}\right) / \mathrm{d} u^{*}\right| \ll\left|\mathrm{d}\left\{f_{\infty}\left(u^{*}\right)-g \mu^{*}\right\} / \mathrm{d} \mu^{*}\right| .
$$

Inserting (41) and (42) and performing the differentiations with the aid of (14) one obtains

$$
\begin{aligned}
&(2 V)^{-1}\left|g\left(\frac{1}{2}, u^{*}\right)\right|^{-1}\left|g\left(-\frac{1}{2}, \mu^{*}\right)\right| \\
& \ll \pi^{3 / 2} 2^{-3} V_{T}^{-1}\left|u^{*}\right|\left|g\left(\frac{1}{2}, u^{*}\right)\right| .
\end{aligned}
$$

For small $\left|\mu^{*}\right|=-\mu^{*}$ the functions $g\left(\frac{1}{2}, \mu^{*}\right)$ and $g\left(-\frac{1}{2}\right.$, $\left.\mu^{*}\right)$ are approximately given by

$$
\left.\begin{array}{l}
g\left(\frac{1}{2}, \mu^{*}\right)=\pi^{\frac{1}{2}}\left(-\mu^{*}\right)^{-\frac{1}{2}} \\
g\left(-\frac{1}{2}, \mu^{*}\right)=\frac{1}{2} \pi^{\frac{1}{2}}\left(-\mu^{*}\right)^{-3 / 2}
\end{array}\right\}
$$

which are the dominant contributions for $n= \pm \frac{1}{2}$ from the general asymptotic expansion for small $-\mu^{*}$,

$g\left(n, \mu^{*}\right)=\Gamma(1-n)\left(-\mu^{*}\right)^{n-1}+\sum_{l=0}^{\infty} \frac{\zeta(n-l)}{l !}\left(\mu^{*}\right)^{l}$,

given by Robinson ${ }^{10}$ for non-integer $n$.

Hence a numerical factor neglected,(44) is found equivalent to

$$
V_{T} / V \ll\left(-\mu^{*}\right)^{3 / 2} \quad \text { or } \quad\left(V_{T} / V\right)^{2 / 3} \ll\left(-\mu^{*}\right) .
$$

This again is condition (39) on account of the definitions of $V_{T}$ and $\varepsilon_{100}$ given in (8) and (15), respectively ${ }^{11}$.

Approximation 3): This approximation consists in replacing the argument of the expontential under the integral (27) by the first two terms of the power series

10 J. E. Robinson, Phys. Rev. 83, 678 [1951].

11 By the way we denote here the leading term in the asymtotic expansion of $f(Q)$ for small values of $Q_{c}-Q$. This is $f(\varrho) \approx\left(16 / 3 \pi^{4}\right) V_{T}{ }^{2} k T\left(Q_{\mathrm{c}}-\varrho\right)^{3}$ as may be seen
(33). This is a god approximation if the exponential falls off to negligible values within an interval around the saddle-point which is small compared to the radius of convergency, $r$.

This condition may be expressed by

$$
V \psi^{\prime \prime}(\xi) r^{2} \gg 1 \text {. }
$$

The radius of convergency is given by

$$
r=1-\xi \approx-u^{*}
$$

for small values of $-u^{*}$. Inserting this into (48) and multiplying the left-hand side by $\Sigma^{2}$, which is near to 1 , one obtains:

$$
V \xi^{2}, y^{\prime \prime}(\xi)\left(-!^{*}\right) \geq \gg 1 \text {. }
$$

The left-hand side may again be estimated by inserting the thermodynamic limit of the corresponding quantities. With the aid of (35), (12), (14), and (45) one finally again arrives at (47) or, equivalently, (39). In condition (47), $-\mu *$ may be expressed by the deviation $\varrho_{c}-\varrho$ of the density from the critical density $\varrho_{c}$. The relevant relations for this purpose are (11), (12), (14), (7), and the asymptotic expansion (46) for $n=$ $3 / 2$. Retaining only the leading term for small $-\mu^{*}$ or $\varrho_{c}-\varrho$ respectively and again neglecting an unimportant numerical factor, one obtains (47) in the form

$$
V_{T} / V \ll\left(Q_{\mathrm{c}}-\underline{o}\right)^{3} / Q_{\mathrm{c}}{ }^{3}
$$

which is the same as (17b) stated in Section I.

After having reached the aim of this section it seems worthwhile to review the simple geometrical meaning which conditions (16a) and (39) receive in Fig. 1 above: Both conditions regard the density of poles on the real axis $z \geqslant 1$ which correspond to the singleparticle states. The first of these conditions postulates that the distance of neighbouring poles must be small compared to one. According to the second this distance must, moreover, be small compared to the distance $1-\xi \approx-\mu^{*}$ of the saddle-point from the pole corresponding to the single-particle ground-state. It is only when both conditions are fullfilled that the discrete spectrum of poles may be approximated by a continuum in the calculation of the free energy and its derivatives in the case $\varrho<\varrho_{\mathrm{c}}$.

by inserting the expansion (46) into (12) (11) and (9) and expressing $\left(-\mu^{*}\right)$ by $\varrho_{c}-\varrho$ via (11). This formula wasused in Eqn. (24) of Ref. ${ }^{4}$ without detailed derivation. 


\section{The Condensed System}

The thermodynamic free energy is a good approximation to $f_{V}(L)$ not only in the case $1-\xi \gg \varepsilon_{100} / k T$ discussed in the preceding section but also for the case $1-\xi \ll \varepsilon_{100} / k T$, that means for the saddle-point lying very close to the $\boldsymbol{k}=0$ pole indicated in Figure 1. In this case $f_{V}(L)$ becomes nearly independent of $L$ on account of Bose condensation into the ground-state $\boldsymbol{k}=0$.

The method of steepest descend is not applicable to our problem in this case since condition (48) fails now. There has been some doubt as to the results of the method even in the thermodynamic limit many years ago 12.13.14. These doubts are meanwhile removed by several authors careful investigations ${ }^{15}, 16.17$, which, essentially, proved that in the thermodynamic limit the results obtained by a formal application of lowest order saddle-point integration did not differ from rigorous results obtained by other means. For the present investigation on the finite system, however, a method different from saddle-point integration has to be developped.

Our aim is the proof that far enough from the critical density, $f_{\mathrm{I}}\left(V_{\varrho}\right)$ as a function of $\varrho$ approximately has the same property as $f(\varrho)$, namely: to be independent of $\varrho$. In fact for the finite system in contrast with the thermodynamic limit aways

$$
f_{1}\left(L_{1}\right)-f_{1} \cdot\left(L_{2}\right)>0 \quad \text { (52) for } L_{2}>L_{1}
$$

holds. This follows from (66) below in combination with (6). The content of this section will be to show that the difference on the left-hand side of (52) becomes very small for $L_{1} / V=\varrho$ satisfying (18a). More definitely it will be shown that

$$
\begin{array}{r}
f_{V}\left(L_{1}\right)-f_{V}\left(L_{2}\right)<k T L_{1} / V \cdot \frac{2 k T}{\varepsilon_{100}\left(L_{1}-\tilde{L}_{\mathrm{c}}\right)} \\
\cdot \exp \left[-\frac{\star_{100}\left(L_{1}-\tilde{L}_{\mathrm{c}}\right)}{2 k T}\right]
\end{array}
$$

holds for

$$
L_{2}>L_{1}>\tilde{L}_{\mathrm{c}}
$$

where

$$
\tilde{L}_{\mathrm{c}}=V \underline{\varrho}_{\mathrm{c}}
$$

is the smallest integer larger than or equal to

12 G. Schubert, Z. Naturforsch. 1, 113 [1946]; 2a, 250 [1947].

13 R. B. Dingle, Proc. Cambridge Phil.Soc. 45, 275 [1949].

14 H. N. V. Temperley, Proc. Roy. Soc. London A 199, 361 [1949].

$$
\begin{aligned}
& \tilde{\tilde{L}}_{\mathrm{c}}=6 k T / \varepsilon_{100}+\sum_{\substack{\boldsymbol{k} \neq 0, \varepsilon_{k} \neq \varepsilon_{100}}}\left\{\exp \left[\left(\varepsilon_{k}-\varepsilon_{100}\right) / k T\right]-1\right\}^{-1}, \\
& \text { i. e. } \quad \tilde{L}_{\mathrm{c}}=\left[\tilde{\tilde{L}}_{\mathrm{c}}\right]
\end{aligned}
$$

in a conventional notation.

In fact $\tilde{g}_{\mathrm{c}}$ defined by $(56)$ and $(57 \mathrm{a}, \mathrm{b})$ is easily seen to have the property

$$
\lim _{V \rightarrow \sim} \tilde{g}_{\mathrm{c}}=\underline{o}_{\mathrm{c}}
$$

claimed in Section I. The proof is trivial and omitted for brevity.

Inequality (54) just implies the property of the free energy which was stated in Section I: Assume (18a) to hold for $\varrho=L_{1} / V$. Then the exponent in (54) is large and negative and hence the variation of $f_{V}\left(L_{2}\right)$ with $L_{2}$ is negligibly small. In other words: the finite system free energy is practically constant for Boson numbers above $L_{1}$ in agreement with the thermodynamic free energy.

The rest of this section will be devoted to the formal mathematical proof of (54). For this purpose let

$$
Z^{\prime}(\lambda)=(2 \pi i)^{-1} \oint z^{-i} \exp \left[V \varphi^{\prime}(z)\right] \mathrm{d} z / z
$$

denote the partition function of a finite Bose system differing from the previous one by the omission of just the $\boldsymbol{k}=0$ single-particle state. $\lambda \geqslant 0$ is the number of Bosons. We mark all quantities belonging to the new system by setting a prime to the corresponding symbol and, moreover, indicate by a prime the absence of the index $\boldsymbol{k}=0$ in sums or products over $\boldsymbol{k}$.

$\exp \left[V \varphi^{\prime}(z)\right]=\underset{\lambda}{\sum} Z^{\prime}(\lambda) z^{\lambda}=\underset{\boldsymbol{k}}{I I^{\prime}}\left[1-z \exp \left(\varepsilon_{\boldsymbol{k}} / k T\right)\right]^{-1}$

is the generating function. The saddle-point $\xi^{\prime}(L)$ of the function $\exp \left[V \varphi^{\prime}(z)\right] / z^{L}$ is given as a function of $L$ by

$$
L=\underset{\boldsymbol{k}}{\sum^{\prime}}\left[\exp \left(\varepsilon_{\boldsymbol{k}} / k T\right) / \xi^{\prime}(L)-1\right]^{-1} .
$$

Two properties of the function $\xi^{\prime}(L)$ will be of use later, namely

$\mathrm{d} \xi^{\prime}(L) / \mathrm{d} L>1 \quad(62) \quad$ and $\quad \xi^{\prime}(L)<\exp \left(\varepsilon_{100} / k T\right) .(63)$

15 A. R. Fraser, Phil. Mag. (7) 42, 156 and 165 [1951].

${ }_{16}$ R. B. Dingle, Adv. Phys. (Phil. Mag. Suppl.) 1, 111 [1952].

17 P. T. Landsberg, Proc. Cambridge Phil. Soc. 50, 65 [1954]. 
Moreover, let $L_{0}$ be defined by

$$
L_{0}=\sum_{\boldsymbol{k}}^{\prime}\left[\exp \left(\varepsilon_{\boldsymbol{k}} / k T\right)-1\right]^{-1},
$$

then

holds.

$$
\xi^{\prime}\left(L_{0}\right)=1
$$

The connection between the partition functiones for the primed and original systems is given by

$$
Z(L)=\sum_{\lambda \leq L} Z^{\prime}(\lambda)
$$

This relations follows from the observation that the whole of distributions of $L$ occupation numbers with a fixed number $L-i \geq 0$ of bosons in the $\boldsymbol{k}=0$ state yields the contribution $\dot{Z}^{\prime}(\hat{\lambda})$ to $Z(L)$.

The condition for $\xi^{\prime}(L)$ being saddle-point

$$
\left[\frac{\mathrm{d}}{\mathrm{d} z}\left\{e q^{\prime}(z) / z^{L}\right\}\right]_{z=\xi^{\prime}(L)}=0
$$

has the consequence

$$
\sum_{i}(\lambda-L)\left[\xi^{\prime}(L)\right]^{\lambda} Z^{\prime}(\lambda)=0
$$

as may be seen by inserting (60) into (67). (68) may be rewritten as

$$
\sum_{\lambda>L}(i-L)\left[\xi^{\prime}(L)\right]^{\lambda} Z^{\prime}(\lambda)=\sum_{\lambda \leqslant L}(L-\lambda)\left[\dot{\xi^{\prime}}(L)\right]^{\lambda} Z^{\prime}(\lambda)
$$

where now all contributions to the sums are non-negative. Let now be

$$
l>L>L_{0}
$$

from which follows, according to (65) and (62),

$$
\xi^{\prime}(l)>\xi^{\prime}(L)>1 \text {. }
$$

Then one may weaken (69) to obtain an inequality by replacing the sum on the left-hand side by the single term $\lambda=l$ and by replacing on the right-hand side the factors $(L-\lambda)\left[\xi^{\prime}(L)\right]^{\lambda}$ by the larger quantities $L\left[\xi^{\prime}(L)\right]^{L}$. Using (66) and re-arranging terms one arrives at

$Z^{\prime}(l) / Z(L)<\frac{L}{l-L}\left[\xi^{\prime}(L)\right]^{-(l-L)}$.

Next an estimate of $\xi^{\prime}(L)$ is needed. For this purpose introduce the sum

$$
\hat{L}=\sum_{\varepsilon_{\boldsymbol{k}} \neq \varepsilon_{100}}^{\prime}\left\{\exp \left[\left(\varepsilon_{\boldsymbol{k}}-\varepsilon_{100}\right) / k T\right]-1\right\}^{-1}
$$

where now not only the ground-state is excluded from the summation but also the three-fold degenerate first excited state. From (61) and (63) follows:

$$
\text { or } \begin{gathered}
L<3\left[\exp \left(\varepsilon_{100} / k T\right) / \xi^{\prime}(L)-1\right]^{-1}+\hat{L} \\
{\left[\xi^{\prime}(L)\right]^{-1}<\left[\exp \left(-\varepsilon_{100} / k T\right)\right][1+3 /(L-\hat{L})]}
\end{gathered}
$$

Now suppose not only (70) but, moreover,

$$
L \geqslant \tilde{L}_{\mathrm{c}} \text {. }
$$

In fact this postulate is stronger than $L>L_{0}$ since $\tilde{L}_{\mathrm{c}}>L_{0}$, as may be easily deduced from the definitions (57) and (64). From (76), (73), and (57) the relation

$$
L-\hat{L} \geqslant 3 \cdot 2 k T / \varepsilon_{100}
$$

is easily deduced. Finally (77) implies the weaker inequality

$$
L-\hat{L}>3 /\left[\exp \left(\varepsilon_{100} / k T\right)-1\right]
$$

which one may combine with (75) to obtain the desired estimate for $\xi^{\prime}(L)$, namely

$$
\left[\xi^{\prime}(L)\right]^{-1}<\exp \left(-\varepsilon_{100} / 2 k T\right) .
$$

The proof of (54) now proceeds as follows: let $L_{1}, L_{2}$ be numbers such that

$$
L_{2}>L_{1}>L \geqslant \tilde{L}_{\mathrm{c}}>L_{0} \text {. }
$$

From (66) one has:

$Z\left(L_{2}\right) / Z\left(L_{1}\right)=1+\sum_{1=L_{1}+1}^{L_{2}} Z^{\prime}(l) / Z\left(L_{1}\right)<1+\sum_{i=L_{1}+1}^{L_{2}} Z^{\prime}(l) / Z(L)$.

This may be transformed to a weaker inequality with the aid of (72) and (79). The result is:

$Z\left(L_{2}\right) / Z\left(L_{1}\right)<1+\frac{L_{1}}{L_{1}-L_{1=}} \sum_{L_{1}+1}^{L_{2}} \exp \left[-\frac{\varepsilon_{100}(l-L)}{2 k T}\right]$.

Besides in deducing (82) use was made of the inequality

$$
L /(l-L)<L_{1} /\left(L_{1}-L\right)
$$

valid for $l>L_{1}>L$.

The sum over $l$ in (82) is easily shown to be smaller than

$$
\left(2 k T / \varepsilon_{100}\right) \exp \left[-\varepsilon_{100}\left(L_{1}-L\right) / 2 k T\right] .
$$

Hence

$$
\frac{Z\left(L_{2}\right)}{Z\left(L_{1}\right)}<1+\frac{L_{1} \quad 2 k T}{L_{1}-L \quad \varepsilon_{100}} \quad \exp \left[-\frac{\varepsilon_{100}\left(L_{1}-L_{.}\right)}{2 k T}\right]
$$

This inequality remains true for $L=\tilde{L}_{\mathrm{c}}$. Inserting this value, taking the logarithm, and weakening the re- 
sulting inequality once more with the aid of the relation $\log (1+x)<x$, one obtains inequality (54). Of course, (6) has to be used also. The proof of (54) was just the aim of this section.

\title{
Dissociative Excitation Processes in $\mathrm{H}_{2} \mathrm{O}$
}

\author{
N. Böse and W. SROKA \\ Institut für Angewandte Physik der Universität Hamburg, Germany \\ (Z. Naturforsch. 28a, 22-26 [1973]; received 5 October 1972)
}

\begin{abstract}
In a single step process the $\mathrm{H}_{2} \mathrm{O}$ molecule is dissociated into excited atoms or ions. The radiation of these particles - $\mathrm{H}^{*}$, O I and O II - is investigated by means of VUV spectroscopy. The excitation functions of the various lines show a pronounced structure. An attempt is made to explain these structures by different excitation processes.
\end{abstract}

Apart from two investigations of the Ly $\alpha$ line at $1215 \AA^{1,2}$ there are no other publications about the radiation in the wavelength region $500-1250 \AA$ caused by dissociative excitation processes from electron collisions in water vapour.

The Lyman series, oxygen atom and oxygen ion lines were investigated. Many structures in the excitation functions were found and an attempt is made to explain these structures by different processes leading to radiation of the same wavelength.

\section{Apparatus}

The apparatus: the electron-molecule collision chamber, the electronic equipment and the vacuumultraviolet-monochromator is described in ${ }^{3}$.

In these experiments the intensity of the radiation of different lines was measured in dependence on the electron energy with a multichannel analyzer. The electron energy scale (number of channel) is calibrated by measuring the appearance potentials of some strong atomic lines in the rare gases. The accuracy in the range $12-23 \mathrm{eV}$ amounts to $\pm 0.5 \mathrm{eV}$ because there are many calibration points, whereas at about $50 \mathrm{eV}$ it is only $\pm 2.0 \mathrm{eV}^{4}$. Special care was taken to purify the water vapour ${ }^{4}$.

Reprint requests to Dr. W. SROKA, Institut für Angegewandte Physik der Universität Hamburg, D-2000 Hamburg, Jungiusstraße 11.

1 J. W. McGowan, J. F. Williams, and D. A. Vroom, Chem. Phys. Letters 3, 614 [1969].

\section{Spectrum}

Figure 1 shows a spectrum obtained by electron bombardment of $\mathrm{H}_{2} \mathrm{O}$ molecules at a pressure of $10^{-3}$ Torr and a beam current of $400 \mu \mathrm{A}$. The electron energy amounted to $150 \mathrm{eV}$.

Six lines of the Lyman series, three O I lines and five oxygen ion lines were observed. It should be noted that the cross sections of the O II lines are almost of the same order of magnitude as those of the $\mathrm{O} I$ lines. Near $600 \AA$ some very weak lines can be seen. Accordingly this part of the spectrum was measured with increased pressure and current and a longer integration time of the rate meter. In this case the signal to noise ratio was about $1 / 2$.

\section{Excitation Processes}

\section{a) Excitation of the Lyman series}

The experiment covered six transitions in the Lyman series. All excitation functions show structures near the threshold. In the shape of the excitation function of Ly $\alpha$ there are at least three different onsets, probably even four. At about $40 \mathrm{eV}$ there seems to be a resonance-like structure.

The other Lyman transitions show only two onsets. We probably can not see any others because of the lower intensity.

2 K. D. Beyer and K. H. Welge, Z. Naturforsch. 22a, 1161 [1967].

3 N. Böse and W. SROKA, Z. Naturforsch. 26a, 1491 [1971].

4 N. BösE, Diplomarbeit, Hamburg 1972. 